\title{
Sliding mode formation tracking control of a tractor and trailer - car system
}

\author{
Fabio Morbidi \\ Dipartimento di Ingegneria dell'Informazione \\ University of Siena \\ Via Roma 56, 53100 Siena, Italy \\ Email: morbidi@dii.unisi.it
}

\author{
Domenico Prattichizzo \\ Dipartimento di Ingegneria dell'Informazione \\ University of Siena \\ Via Roma 56, 53100 Siena, Italy \\ Email: prattichizzo@dii.unisi.it
}

\begin{abstract}
In this paper a new leader-follower formation of nonholonomic mobile robots is studied. The follower is a car-like vehicle and the leader is a tractor pulling a trailer. The leader moves along assigned trajectories and the follower is to maintain a desired distance and orientation to the trailer. A sliding mode control scheme is proposed for asymptotically stabilizing the vehicles to a time-varying desired formation. The attitude angles of the follower and the tractor are estimated via global exponential observers based on the invariant manifold technique. Simulation experiments illustrate the theory and show the effectiveness of the proposed formation controller and nonlinear observers.
\end{abstract}

\section{INTRODUCTION}

Last years have seen a growing interest on control of formations of autonomous robots. This trend has been supported by the recent technological advances on computation and communication capabilities and by the observation that multiagent systems can perform tasks beyond the ability of individual vehicles. By formation control we simply mean the problem of controlling the relative position and orientation of the robots in a group while allowing the group to move as a whole. In this respect, research dealt with ground vehicles [9], [11], [18], surface and underwater autonomous vehicles (AUVs) [10], [12], unmanned aerial vehicles (UAVs) [4], [16], microsatellite clusters [2], [24].

One of the most important approaches to formation control is leader-following. A robot of the formation, designed as the leader, moves along a predefined trajectory while the other robots, the followers, are to maintain a desired posture (distance and orientation) to the leader.

Leader-follower architectures are known to have poor disturbance rejection properties. In addition, the over-reliance on a single agent for achieving the goal may be undesirable especially in adverse conditions. Nevertheless this approach is extremely simple since a reference trajectory is clearly defined by the leader and the internal formation stability is induced by stability of the individual vehicles' control laws.

The following common features can be identified in the literature concerning leader-follower formation control of mobile robots:

Kinematic models: Unicycle models are the most common in the literature [6], [29]. Car-like vehicles are frequent in papers dealing with vehicular systems and control of automated road vehicles (see, e.g. [22] and the references therein).

Formation shape: Rigid formations (i.e., formations where the inter-vehicle desired parameters are constant in time) are frequent in the literature (see the references above). Switching between different rigid formations has been studied recently in [7], [25].

Formation control: The most common formation control strategies are feedback linearization [7], [20], dynamic feedback linearization [19], [30], backstepping [17].

State estimation: The state of the formation is frequently supposed to be known. Otherwise standard nonlinear observers are used: e.g. the extended Kalman filter [7], [20] and recently the unscented Kalman filter [19].

In this paper a new challenging leader-follower formation of nonholonomic mobile robots is studied. The follower is a car-like vehicle and the leader is a multibody mobile robot: a tractor (a car-like vehicle) pulling a trailer. The leader moves along an assigned trajectory while the follower is to maintain a desired distance and orientation to the trailer.

The choice of this specific formation was motivated by a possible real-world application: the control of truck-trailer / car platoons in automated highway systems (AHSs) [1], [26]. Besides the applicative example, it is expected that the proposed multi-robot scheme could be of theoretical interest in nonholonomic systems research.

During the last three decades since [27], variable structure systems (VSS) and sliding mode control (SMC) (that plays a dominant role in VSS theory), have attracted the control research community worldwide (see e.g., [8], [14], [21] and the references therein). One of the distinguishing features of sliding mode is the discontinuous nature of the control action whose primary function is to switch between two different system structures such that a new type of system motion, called sliding mode, exists in a manifold. This peculiar characteristic results in excellent system performance which includes insensitivity to parametric uncertainty and external disturbances.

Sliding mode control has been largely used in the industrial electronic area (induction motors, electric drives [28]). Recently, it has been applied to trajectory tracking of nonholonomic vehicles [5], [31]. Nevertheless, only few 
papers [13], [23], dealt with sliding mode control for robot formations.

In the present paper we propose a globally asymptotically stable sliding mode formation tracking controller for the tractor and trailer - car system. Differently from the literature, the desired leader-follower formation is allowed to vary in time arbitrarily. Actually, any continuous distance and orientation functions can be used to define the formation shape.

Finally, according to the invariant manifold technique proposed in [15], global exponential observers of the attitude angle of the follower and the tractor are designed. These observers are among the first applications of the theory developed by Karagiannis and Astolfi. They revealed simple to implement and they exhibited good performances in the simulation experiments, thus confirming that the invariant manifold technique is a viable alternative to standard nonlinear observer design strategies.

The rest of the paper is organized as follows. Section II is devoted to the problem formulation. In Section III the sliding mode formation tracking controller is designed. In Section IV the nonlinear observers of the attitude angles are presented. In Section V simulation experiments with noisy data illustrate the theory and show the closed-loop system performance. In Section VI the major contributions of the paper are summarized and future research lines are highlighted.

Notation: The following notation is used through the paper: $\mathbb{R}^{+}=\{x \in \mathbb{R} \mid x>0\}, \mathbb{R}_{0}^{+}=\{x \in \mathbb{R} \mid x \geq 0\}$.

$\forall x \in \mathbb{R}, \operatorname{sign}(x)=1$ if $x>0, \operatorname{sign}(x)=0$ if $x=0$ and $\operatorname{sign}(x)=-1$ if $x<0$.

$\forall \mathbf{s}=\left(s_{1}, s_{2}\right)^{T} \in \mathbb{R}^{2}, \operatorname{sign}(\mathbf{s})=\left(\operatorname{sign}\left(s_{1}\right), \operatorname{sign}\left(s_{2}\right)\right)^{T}$, $\|\mathbf{s}\|=\sqrt{s_{1}^{2}+s_{2}^{2}}, \operatorname{diag}\left(s_{1}, s_{2}\right)=\left(\begin{array}{cc}s_{1} & 0 \\ 0 & s_{2}\end{array}\right)$.

\section{PROBLEM FORMULATION}

The leader-follower setup considered in the paper is presented in Fig. 1. The follower $F$ is a car with rear wheels aligned with the vehicle and front wheels allowed to spin about the vertical axis. The kinematic model is,

$$
\left\{\begin{array}{l}
\dot{x}_{F}=v_{F} \cos \theta_{F} \\
\dot{y}_{F}=v_{F} \sin \theta_{F} \\
\dot{\theta}_{F}=\frac{v_{F}}{\ell_{1}} \tan \alpha_{F} \\
\dot{\alpha}_{F}=\omega_{F}
\end{array}\right.
$$

where $\left(x_{F}, y_{F}\right)$ are the coordinates of the midpoint of the rear driving wheels, $\theta_{F}$ is the angle of the car body with respect to the $x$-axis, $\alpha_{F}$ the steering angle with respect to the car body, $\ell_{1}$ the wheelbase of the car and $v_{F}$ and $\omega_{F}$ are respectively the forward velocity of the rear wheels and the steering velocity of the car. The leader $L$ is an articulated vehicle, a tractor (car) pulling a trailer.

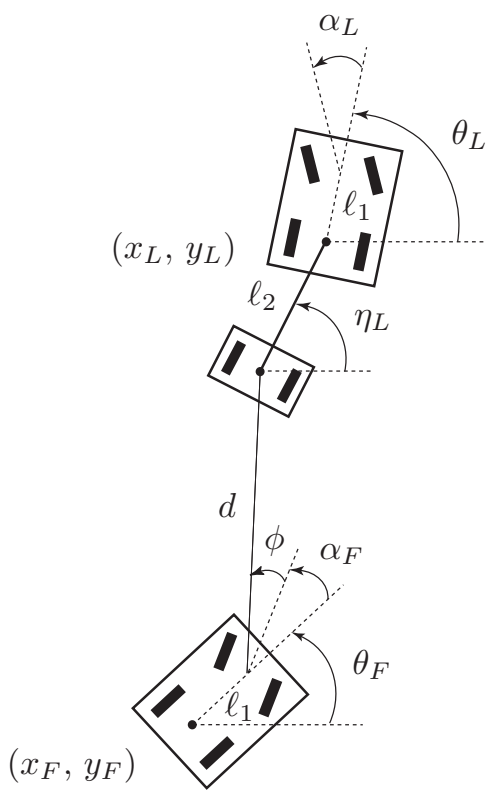

Fig. 1. Leader-follower setup.

The kinematic model is,

$$
\left\{\begin{array}{l}
\dot{x}_{L}=v_{L} \cos \theta_{L} \\
\dot{y}_{L}=v_{L} \sin \theta_{L} \\
\dot{\theta}_{L}=\frac{v_{L}}{\ell_{1}} \tan \alpha_{L} \\
\dot{\eta}_{L}=\frac{v_{L}}{\ell_{2}} \sin \left(\theta_{L}-\eta_{L}\right) \\
\dot{\alpha}_{L}=\omega_{L}
\end{array}\right.
$$

where $x_{L}, y_{L}, \theta_{L}$ and $\alpha_{L}$ are the car part variables of the leader, $\eta_{L}$ is the angle of the trailer with respect to the $x$-axis and $\ell_{2}$ is the distance between the rear wheels of the tractor and the wheels of the trailer. Hereafter we will assume that:

Hypothesis 1: The steering angle of the vehicles is bounded, namely $\alpha_{F}, \alpha_{L} \in[-\pi / 3, \pi / 3]$.

Hypothesis 2: The possible configurations of the tractor and trailer are restricted by a mechanical stop on the revolute joint connecting the two bodies, hence $\eta_{L} \in(0, \pi)$.

With reference to Fig. 1, the following definition introduces the notion of leader-follower formation used in this paper.

Definition 1: Let $d(t): \mathbb{R}_{0}^{+} \rightarrow \mathbb{R}^{+}, \phi(t): \mathbb{R}_{0}^{+} \rightarrow$ $(-\pi / 2, \pi / 2)$ and $d(t), \phi(t) \in \mathcal{C}^{1}$, be two given functions. We say that $L$ and $F$ make a $(d, \phi)$-formation with leader $L$ at time $t$, if,

$$
\begin{gathered}
\left(\begin{array}{c}
x_{L}-\ell_{2} \cos \eta_{L} \\
y_{L}-\ell_{2} \sin \eta_{L}
\end{array}\right)-\left(\begin{array}{c}
x_{F}+\ell_{1} \cos \theta_{F} \\
y_{F}+\ell_{1} \sin \theta_{F}
\end{array}\right)= \\
=d\left(\begin{array}{c}
\cos \left(\theta_{F}+\alpha_{F}+\phi\right) \\
\sin \left(\theta_{F}+\alpha_{F}+\phi\right)
\end{array}\right)
\end{gathered}
$$


and, simply, that $L$ and $F$ make a $(d, \phi)$-formation (with leader $L$ ) if (3) holds for any $t \geq 0$.

$d$ is the distance between the midpoint of the wheels of the trailer and the midpoint of the front wheels of the follower and $\phi$ is the visual angle of the trailer from the follower, minus the steering angle $\alpha_{F}$ (see Fig. 1).

Note that in order to avoid collisions between the vehicles, a sufficient condition is that $d(t) \geq 2 \ell_{1}+\ell_{2}$ for any $t \geq 0$.

\section{SLIDING MODE FORMATION TRACKING CONTROL}

In this section we design a sliding mode controller for globally asymptotically stabilizing the vehicles to the desired $(d, \phi)$-formation. We here recall that the leader moves along an assigned trajectory and consequently the vector $\left(v_{L}, \omega_{L}\right)^{T}$ is known.

Proposition 1: According to Hypotheses 1, 2 and Definition 1 , introduce the error vector

$$
\begin{gathered}
\left(\begin{array}{l}
e_{1} \\
e_{2}
\end{array}\right)=\left(\begin{array}{c}
x_{L}-\ell_{2} \cos \eta_{L} \\
y_{L}-\ell_{2} \sin \eta_{L}
\end{array}\right)-\left(\begin{array}{c}
x_{F}+\ell_{1} \cos \theta_{F} \\
y_{F}+\ell_{1} \sin \theta_{F}
\end{array}\right)- \\
-d\left(\begin{array}{c}
\cos \left(\theta_{F}+\alpha_{F}+\phi\right) \\
\sin \left(\theta_{F}+\alpha_{F}+\phi\right)
\end{array}\right)
\end{gathered}
$$

and define the sliding surfaces

$$
\begin{aligned}
& s_{1}=\dot{e}_{1}+k_{1} e_{1} \\
& s_{2}=\dot{e}_{2}+k_{2} e_{2}
\end{aligned}
$$

where $k_{1}, k_{2}$ are positive constants. The control law

$$
\left(\begin{array}{c}
\dot{v}_{F} \\
\dot{\omega}_{F}
\end{array}\right)=\mathbf{A}^{-1}\left(-\mathbf{B}\left(\begin{array}{c}
v_{L} \\
v_{L}^{2} \\
\dot{v}_{L}
\end{array}\right)-\mathbf{C}\left(\begin{array}{c}
v_{F} \\
\omega_{F} \\
v_{F}^{2} \\
\omega_{F}^{2} \\
v_{F} \omega_{F}
\end{array}\right)-\mathbf{D}-\mathbf{F} \operatorname{sign}(\mathbf{s})\right)
$$

globally asymptotically stabilizes the sliding surfaces to zero. The matrices $\mathbf{A}, \mathbf{B}, \mathbf{C}, \mathbf{F}$ and the vector $\mathbf{D}$ are defined in the proof given below.

Proof: Define $\lambda_{F}=\theta_{F}+\alpha_{F}+\phi$ and $\gamma_{L}=\theta_{L}-\eta_{L}$.

Let compute $\dot{s}_{1}$ and $\dot{s}_{2}$ from (5),

$$
\dot{s}_{1}=\ddot{x}_{L}+\ell_{2} \cos \eta_{L} \dot{\eta}_{L}^{2}+\ell_{2} \sin \eta_{L} \ddot{\eta}_{L}-\ddot{x}_{F}+\ell_{1} \cos \theta_{F} \dot{\theta}_{F}^{2}
$$$$
+\ell_{1} \sin \theta_{F} \ddot{\theta}_{F}-\ddot{d} \cos \lambda_{F}+2 \dot{d} \sin \lambda_{F} \dot{\lambda}_{F}+d \cos \lambda_{F} \dot{\lambda}_{F}^{2}
$$$$
+d \sin \lambda_{F} \ddot{\lambda}_{F}+k_{1}\left(\dot{x}_{L}+\ell_{2} \sin \eta_{L} \dot{\eta}_{L}-\dot{x}_{F}+\ell_{1} \sin \theta_{F} \dot{\theta}_{F}\right.
$$$$
\left.-\dot{d} \cos \lambda_{F}+d \sin \lambda_{F} \dot{\lambda}_{F}\right) \text {, }
$$

$\dot{s}_{2}=\ddot{y}_{L}+\ell_{2} \sin \eta_{L} \dot{\eta}_{L}^{2}-\ell_{2} \cos \eta_{L} \ddot{\eta}_{L}-\ddot{y}_{F}+\ell_{1} \sin \theta_{F} \dot{\theta}_{F}^{2}$

$-\ell_{1} \cos \theta_{F} \ddot{\theta}_{F}-\ddot{d} \sin \lambda_{F}-2 \dot{d} \cos \lambda_{F} \dot{\lambda}_{F}+d \sin \lambda_{F} \dot{\lambda}_{F}^{2}$

$-d \cos \lambda_{F} \ddot{\lambda}_{F}+k_{2}\left(\dot{y}_{L}-\ell_{2} \cos \eta_{L} \dot{\eta}_{L}-\dot{y}_{F}-\ell_{1} \cos \theta_{F} \dot{\theta}_{F}\right.$

$\left.-\dot{d} \sin \lambda_{F}-d \cos \lambda_{F} \dot{\lambda}_{F}\right)$.
Replacing the derivatives according to (1) and (2) and writing the resulting expression in a matrix form, we obtain,

$$
\dot{\mathbf{s}}=\mathbf{A}\left(\begin{array}{c}
\dot{v}_{F} \\
\dot{\omega}_{F}
\end{array}\right)+\mathbf{B}\left(\begin{array}{c}
\dot{v}_{L} \\
v_{L}^{2} \\
\dot{v}_{L}
\end{array}\right)+\mathbf{C}\left(\begin{array}{c}
v_{F} \\
\omega_{F} \\
v_{F}^{2} \\
\omega_{F}^{2} \\
v_{F} \omega_{F}
\end{array}\right)+\mathbf{D}
$$

where

$$
\mathbf{A}=\left(\begin{array}{cc}
-\cos \theta_{F}+\tan \alpha_{F}\left(\sin \theta_{F}+\frac{d}{\ell_{1}} \sin \lambda_{F}\right) & d \sin \lambda_{F} \\
-\sin \theta_{F}-\tan \alpha_{F}\left(\cos \theta_{F}+\frac{d}{\ell_{1}} \cos \lambda_{F}\right) & -d \cos \lambda_{F}
\end{array}\right) .
$$

The components of the matrices $\mathbf{B} \in \mathbb{R}^{2 \times 3}$ and $\mathbf{C} \in \mathbb{R}^{2 \times 5}$ are

$$
\begin{aligned}
& \mathbf{B}_{11}=k_{1} \cos \left(\theta_{L}-\gamma_{L}\right) \cos \gamma_{L} \\
& \mathbf{B}_{12}=\frac{\tan \alpha_{L}}{\ell_{1}} \cos \left(\theta_{L}-\gamma_{L}\right) \sin \left(2 \theta_{L}-\gamma_{L}\right)+\frac{\sin \gamma_{L}}{\ell_{2}} \sin \left(2 \gamma_{L}-\theta_{L}\right) \\
& \mathbf{B}_{13}=\cos \left(\theta_{L}-\gamma_{L}\right) \cos \gamma_{L} \\
& \mathbf{B}_{21}=k_{2} \sin \left(\theta_{L}-\gamma_{L}\right) \cos \gamma_{L} \\
& \mathbf{B}_{22}=-\frac{\tan \alpha_{L}}{\ell_{1}} \sin \left(\theta_{L}-\gamma_{L}\right) \sin \gamma_{L}+\frac{\sin \gamma_{L}}{\ell_{2}} \cos \left(2 \gamma_{L}-\theta_{L}\right) \\
& \mathbf{B}_{23}=\sin \left(\theta_{L}-\gamma_{L}\right) \cos \gamma_{L}, \\
& \mathbf{C}_{11}=\tan \alpha_{F}\left(\frac{\sin \lambda_{F}\left(2 \dot{d}+k_{1} d\right)+2 d \dot{\phi} \cos \lambda_{F}}{\ell_{1}}+k_{1} \sin \theta_{F}\right)-k_{1} \cos \theta_{F} \\
& \mathbf{C}_{12}=\sin \lambda_{F}\left(2 \dot{d}+d k_{1}\right)+2 d \dot{\phi} \cos \lambda_{F} \\
& \mathbf{C}_{13}=\frac{\sin \theta_{F} \tan \alpha_{F}}{\ell_{1}}+\frac{\tan ^{2} \alpha_{F}}{\ell_{1}^{2}}\left(\ell_{1} \cos \theta_{F}+d \cos \lambda_{F}\right) \\
& \mathbf{C}_{14}=d \cos \lambda_{F} \\
& \mathbf{C}_{15}=\frac{1}{\ell_{1} \cos \alpha_{F}}\left(\ell_{1} \sin \theta_{F}+d \sin \lambda_{F}\right)+\frac{2 d}{\ell_{1}} \cos \lambda_{F} \tan \alpha_{F} \\
& \mathbf{C}_{21}=-\tan \alpha_{F}\left(\frac{\cos \lambda_{F}\left(2 \dot{d}+k_{2} d\right)-2 d \dot{\phi} \sin \lambda_{F}}{\ell_{1}}+k_{2} \cos \theta_{F}\right)-k_{2} \sin \theta_{F} \\
& \mathbf{C}_{22}=-\cos \lambda_{F}\left(2 \dot{d}+d k_{2}\right)+2 d \dot{\phi} \sin \lambda_{F} \\
& \mathbf{C}_{23}=-\frac{\cos \theta_{F} \tan \alpha_{F}}{\ell_{1}}+\frac{\tan ^{2} \alpha_{F}}{\ell_{1}^{2}}\left(\ell_{1} \sin \theta_{F}+d \sin \lambda_{F}\right) \\
& \mathbf{C}_{24}=d \sin \lambda_{F} \\
& \mathbf{C}_{25}=\frac{1}{\ell_{1} \cos \alpha_{F}}\left(\ell_{1} \cos \theta_{F}-d \cos \lambda_{F}\right)+\frac{2 d}{\ell_{1}} \sin \lambda_{F} \tan \alpha_{F} \\
& \mathbf{t a n}_{F}
\end{aligned}
$$

and the vector $\mathbf{D}$ is given by,

$$
\mathbf{D}=\left(\begin{array}{cc}
-\ddot{d}+d \dot{\phi}^{2}-k_{1} \dot{d} & 2 \dot{d} \dot{\phi}+d \ddot{\phi}+k_{1} d \dot{\phi} \\
-2 \dot{d} \dot{\phi}-d \ddot{\phi}-k_{2} d \dot{\phi} & -\ddot{d}+d \dot{\phi}^{2}-k_{1} \dot{d}
\end{array}\right)\left(\begin{array}{c}
\cos \lambda_{F} \\
\sin \lambda_{F}
\end{array}\right) .
$$

Consider the candidate Lyapunov function

$$
V=\frac{1}{2} \mathbf{s}^{T} \mathbf{s}
$$

whose derivative along the system trajectories is

$$
\dot{V}=\mathbf{s}^{T}\left(\mathbf{A}\left(\begin{array}{c}
\dot{v}_{F} \\
\dot{\omega}_{F}
\end{array}\right)+\mathbf{B}\left(\begin{array}{c}
\dot{v}_{L} \\
v_{L}^{2} \\
\dot{v}_{L}
\end{array}\right)+\mathbf{C}\left(\begin{array}{c}
v_{F} \\
\omega_{F} \\
v_{F}^{2} \\
\omega_{F}^{2} \\
v_{F} \omega_{F}
\end{array}\right)+\mathbf{D}\right) .
$$


Substituting the control law (6) in (7), with $\mathbf{F}=\operatorname{diag}\left(f_{1}, f_{2}\right)$, $f_{1}, f_{2} \in \mathbb{R}^{+}$, we obtain,

$$
\dot{V}=-f_{1}\left|s_{1}\right|-f_{2}\left|s_{2}\right|
$$

that is strictly less than zero for all $\mathbf{s} \neq \mathbf{0}$. Therefore (6) globally asymptotically stabilizes the sliding surfaces to zero.

Remark 1: The proposed control law (6) becomes singular when

$$
\operatorname{det}(\mathbf{A})=\frac{d \cos \phi}{\cos \alpha_{F}}=0 .
$$

From Hypothesis 1 and the assumptions in Definition 1, $\operatorname{det}(\mathbf{A}) \neq 0$ for any $t \geq 0$, and consequently the proposed control law has no singularities.

Remark 2: Note that since control (6) explicitly depends on trailer's parameters (i.e., $\ell_{2}, \eta_{L}$ ), the trajectory of the follower does not coincide in general with that of car following another car (the tractor). The two paths become similar when the curvature of leader's trajectory is small or the trailer is rigidly connected to the tractor.

\section{OBSERVERS DESIGN}

In this section, according to the invariant manifold technique introduced in [15], we design global exponential observers of the attitude angles $\theta_{F}$ and $\theta_{L}$ based on the measurement vectors $\left(x_{F}, y_{F}, \alpha_{F}\right)^{T}$ and $\left(x_{L}, y_{L}, \alpha_{L}\right)^{T}$, respectively.

An alternative observer of $\theta_{L}$ only based on $\gamma_{L}=\theta_{L}-\eta_{L}$, the angle between the cable connecting the tractor to the trailer and the trailer longitudinal axis, is also proposed.

\section{A. Estimation of the angle $\theta_{F}$}

Proposition 2: Consider model (1) and assume that the set $\mathcal{Q}=\left\{t \in \mathbb{R}_{0}^{+}: v_{F}(t)=0\right\}$ is finite. Define the system,

$$
\begin{aligned}
\dot{\xi}_{1}= & -\left(\xi_{2}+\Lambda y_{F} v_{F}\right) \frac{v_{F}}{\ell_{1}} \tan \alpha_{F}- \\
& -\Lambda v_{F}^{2}\left(\xi_{1}+\Lambda x_{F} v_{F}\right)-\Lambda x_{F} \dot{v}_{F} \\
\dot{\xi}_{2}= & \left(\xi_{1}+\Lambda x_{F} v_{F}\right) \frac{v_{F}}{\ell_{1}} \tan \alpha_{F}- \\
& -\Lambda v_{F}^{2}\left(\xi_{2}+\Lambda y_{F} v_{F}\right)-\Lambda y_{F} \dot{v}_{F}
\end{aligned}
$$

where $\Lambda$ is a positive gain. Then, for any initial condition $\left(\xi_{1}(0), \xi_{2}(0)\right)^{T}$,

$$
\hat{\theta}_{F}=\arctan \left(\frac{\xi_{2}+\Lambda y_{F} v_{F}}{\xi_{1}+\Lambda x_{F} v_{F}}\right)
$$

exponentially converges to the actual attitude angle $\theta_{F}$. Proof: Define the error variables,

$$
\begin{aligned}
& z_{1}=-\xi_{1}-\Lambda x_{F} v_{F}+\cos \theta_{F} \\
& z_{2}=-\xi_{2}-\Lambda y_{F} v_{F}+\sin \theta_{F} .
\end{aligned}
$$

Their derivatives are,

$$
\begin{aligned}
& \dot{z}_{1}=-\frac{v_{F}}{\ell_{1}} \sin \theta_{F} \tan \alpha_{F}-\dot{\xi}_{1}-\Lambda v_{F}^{2} \cos \theta_{F}-\Lambda x_{F} \dot{v}_{F} \\
& \dot{z}_{2}=\frac{v_{F}}{\ell_{1}} \cos \theta_{F} \tan \alpha_{F}-\dot{\xi}_{2}-\Lambda v_{F}^{2} \sin \theta_{F}-\Lambda y_{F} \dot{v}_{F} .
\end{aligned}
$$

Substituting (8) and (9) in (11), we obtain,

$$
\begin{aligned}
& \dot{z}_{1}=-\frac{v_{F}}{\ell_{1}} z_{2} \tan \alpha_{F}-\Lambda v_{F}^{2} z_{1} \\
& \dot{z}_{2}=\frac{v_{F}}{\ell_{1}} z_{1} \tan \alpha_{F}-\Lambda v_{F}^{2} z_{2} .
\end{aligned}
$$

Define the candidate Lyapunov function,

$$
V=\frac{1}{2}\left(z_{1}^{2}+z_{2}^{2}\right)
$$

whose time derivative along (12) satisfies,

$$
\dot{V}=-2 \Lambda v_{F}^{2} V .
$$

Since $\mathcal{Q}$ is finite, then necessarily there is a time instant $\bar{t}$ such that $\dot{V}(t) \leq-\varepsilon(t) V(t), \varepsilon(t) \in \mathbb{R}^{+}, \forall t>\bar{t}$ and this concludes the proof.

\section{B. Estimation of the angle $\theta_{L}$}

In order to estimate $\theta_{L}$, an analogous observer can be designed by simply referring equations (8), (9), (10) to the leader's kinematics. This observer will be used in the simulation experiments.

An alternative observer of the angle $\theta_{L}$ is here introduced. It is not an exponential estimator of $\theta_{L}$, but since it depends on a single measured variable, $\gamma_{L}$, is possibly less sensitive to disturbances.

Proposition 3: Consider model (2) and the system,

$$
\begin{aligned}
& \dot{\xi}_{3}=-\frac{v_{L}}{\ell_{2}} \xi_{4} \sin \gamma_{L} \\
& \dot{\xi}_{4}=\frac{v_{L}}{\ell_{2}} \xi_{3} \sin \gamma_{L} .
\end{aligned}
$$

Then, for any $\left(\xi_{3}(0), \xi_{4}(0)\right)^{T}$ such that $\hat{\eta}_{L}(0)=\eta_{L}(0)$,

$$
\hat{\theta}_{L}=\gamma_{L}+\arctan \left(\frac{\xi_{4}}{\xi_{3}}\right)
$$

gives an estimate of the angle $\theta_{L}$.

Proof: The proof is analogous to that of Proposition 2.

Define the error variables,

$$
\begin{aligned}
& z_{3}=-\xi_{3}+\cos \eta_{L} \\
& z_{4}=-\xi_{4}+\sin \eta_{L} .
\end{aligned}
$$

Their derivatives are,

$$
\begin{aligned}
& \dot{z}_{3}=-\frac{v_{L}}{\ell_{2}} \sin \eta_{L} \sin \gamma_{L}-\dot{\xi}_{3} \\
& \dot{z}_{4}=\frac{v_{L}}{\ell_{2}} \cos \eta_{L} \sin \gamma_{L}-\dot{\xi}_{4} .
\end{aligned}
$$

Substituting (13) and (14) in (15), we obtain,

$$
\begin{aligned}
& \dot{z}_{3}=-\frac{v_{L}}{\ell_{2}} z_{4} \sin \gamma_{L} \\
& \dot{z}_{4}=\frac{v_{L}}{\ell_{2}} z_{3} \sin \gamma_{L} .
\end{aligned}
$$

Define the candidate Lyapunov function,

$$
V=\frac{1}{2}\left(z_{3}^{2}+z_{4}^{2}\right) .
$$

Its derivative along (16) is $\dot{V}=0$ from which the result follows. 


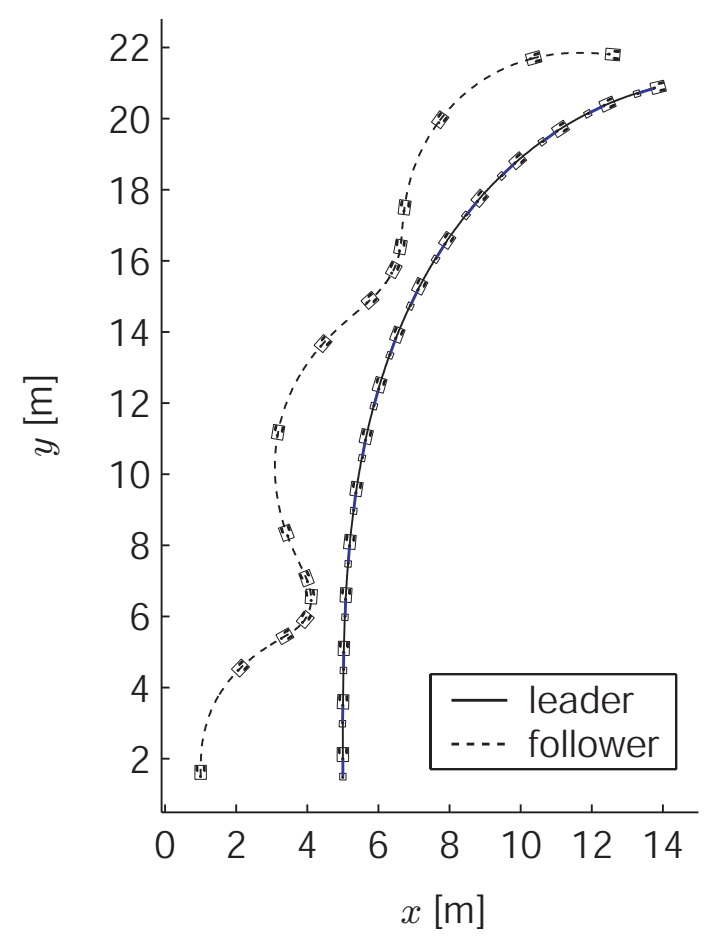

(a)

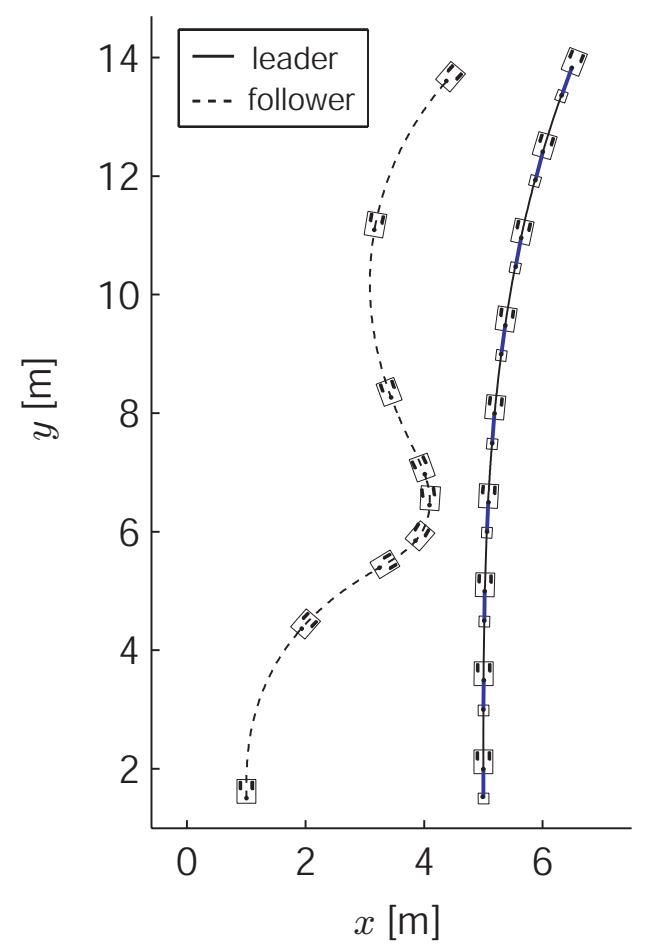

(b)

Fig. 2. Simulation I (ideal measurements): (a) Trajectory of the leader and the follower; (b) Trajectory of the vehicles in the first 8 seconds of the simulation.

Remark 3: Note that the exponential observer of $\theta_{L}$ does not use the angle $\gamma_{L}$. Nevertheless $\gamma_{L}$ needs to be measured in order to compute the sliding surfaces $s_{1}, s_{2}$ and matrix $\mathbf{B}$ in (6). The same conclusions hold for the observer in Proposition 3 and vector $\left(x_{L}, y_{L}, \alpha_{L}\right)^{T}$.

\section{Simulation Results}

Two simulation experiments were carried out to evaluate the performance of the sliding mode controller and the nonlinear observers presented in Sections III and IV. The first simulation refers to the case of ideal measurements. In the second simulation the vectors $\left(x_{F}, y_{F}, \alpha_{F}\right)^{T},\left(x_{L}, y_{L}, \alpha_{L}\right)^{T}$ and $\gamma_{L}$ are supposed to be corrupted by zero mean gaussian noise with variance $0.5 \times 10^{-3}$. The initial conditions of the leader and the follower are, $x_{L}(0)=5 \mathrm{~m}, y_{L}(0)=2 \mathrm{~m}, \theta_{L}(0)=\pi / 2 \mathrm{rad}$, $\alpha_{L}(0)=0 \mathrm{rad}, \eta_{L}(0)=\pi / 2 \mathrm{rad}, x_{F}(0)=1 \mathrm{~m}, y_{F}(0)=$ $1.5 \mathrm{~m}, \theta_{F}(0)=\pi / 2 \mathrm{rad}, \alpha_{F}(0)=0 \mathrm{rad}$.

In order to compute the actuating control input, equation (6) needs to be integrated and some initial values $v_{F}(0), \omega_{F}(0)$ to be fixed. Although the stability of the control does not depend on these values, a common procedure to initialize the velocities in order to get good performances, is the following. Compute the derivative of (4) for $t=0$ and equal it to zero,

$$
\begin{aligned}
\dot{e}_{1}(0) & =v_{L}(0) \cos \eta_{L}(0) \cos \gamma_{L}(0)+v_{F}(0)\left(-\cos \theta_{F}(0)\right. \\
& \left.+\sin \theta_{F}(0) \tan \alpha_{F}(0)+\frac{d(0)}{\ell_{1}} \sin \lambda_{F}(0) \tan \alpha_{F}(0)\right) \\
& +\omega_{F}(0) d(0) \sin \lambda_{F}(0)-\dot{d}(0) \cos \lambda_{F}(0) \\
& +d(0) \dot{\phi}(0) \sin \lambda_{F}(0)=0
\end{aligned}
$$

$$
\begin{aligned}
\dot{e}_{2}(0) & =v_{L}(0) \sin \eta_{L}(0) \cos \gamma_{L}(0)-v_{F}(0)\left(\sin \theta_{F}(0)\right. \\
& \left.+\cos \theta_{F}(0) \tan \alpha_{F}(0)+\frac{d(0)}{\ell_{1}} \cos \lambda_{F}(0) \tan \alpha_{F}(0)\right) \\
& -\omega_{F}(0) d(0) \cos \lambda_{F}(0)-\dot{d}(0) \sin \lambda_{F}(0) \\
& -d(0) \dot{\phi}(0) \cos \lambda_{F}(0)=0 .
\end{aligned}
$$

Solving the above equations with respect to $v_{F}(0), \omega_{F}(0)$ with $\lambda_{F}(0) \in(0, \pi)$, we finally obtain,

$$
\begin{aligned}
v_{F}(0)= & \frac{\cos \alpha_{F}(0)}{\cos \phi(0)}\left(v_{L}(0) \cos \gamma_{L}(0) \cos \left(\lambda_{F}(0)-\eta_{L}(0)\right)-\dot{d}(0)\right) \\
\omega_{F}(0) & =\frac{1}{d(0) \sin \lambda_{F}(0)}\left(-v_{L}(0) \cos \eta_{L}(0) \cos \gamma_{L}(0)\right. \\
& +\frac{v_{L}(0) \cos \gamma_{L}(0) \cos \left(\lambda_{F}(0)-\eta_{L}(0)\right)-\dot{d}(0)}{\cos \phi(0)} \\
& \cdot\left(\cos \left(\alpha_{F}(0)+\theta_{F}(0)\right)-\frac{d(0)}{\ell_{1}} \sin \lambda_{F}(0) \sin \alpha_{F}(0)\right) \\
& \left.+\dot{d}(0) \cos \lambda_{F}(0)-d(0) \dot{\phi}(0) \sin \lambda_{F}(0)\right)
\end{aligned}
$$

We set $\ell_{1}=0.2 \mathrm{~m}, \ell_{2}=0.5 \mathrm{~m}$ and we chose the following parameters for the controller and the observers: $k_{1}=k_{2}=1, f_{1}=f_{2}=2$ and $\Lambda=5, \xi_{1}(0)=\xi_{2}(0)=0$. Table I shows leader's velocity and functions $d(t)$ and $\phi(t)$ used in Simulation I and II. The steering velocity of the leader in Simulation II (see Table I) is $\Omega(t)=\pi / 600$ if $t<3 \mathrm{~s}, \Omega(t)=-\pi / 600$ if $t \geq 3 \mathrm{~s}$. Figs. 2 and 3 are relative to Simulation I. 

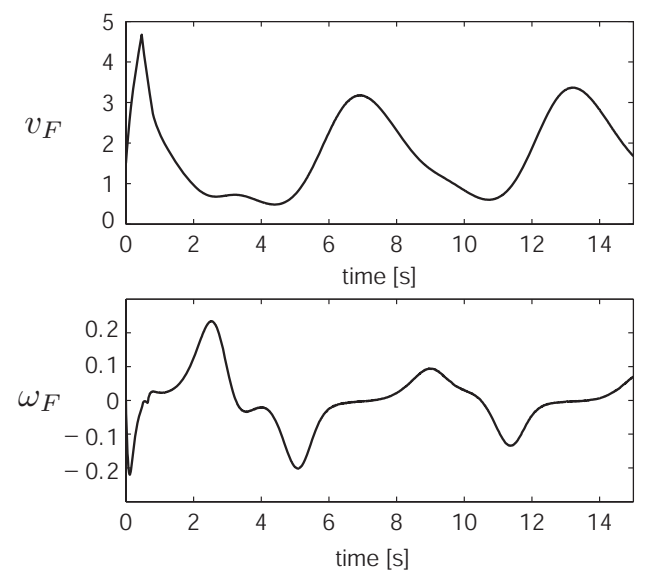

(a)

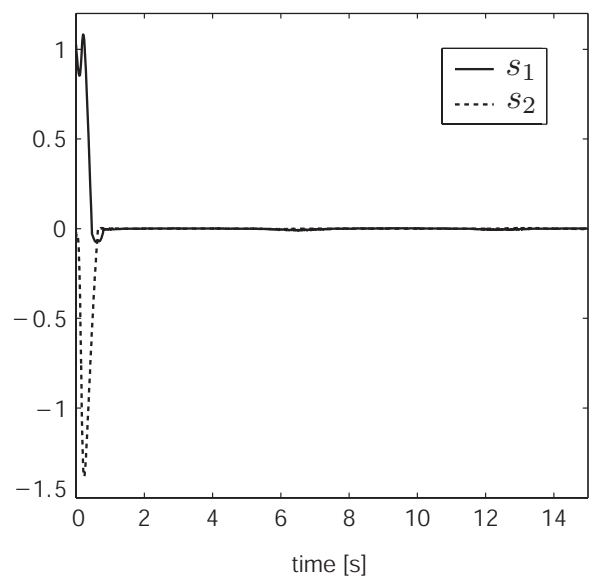

(b)

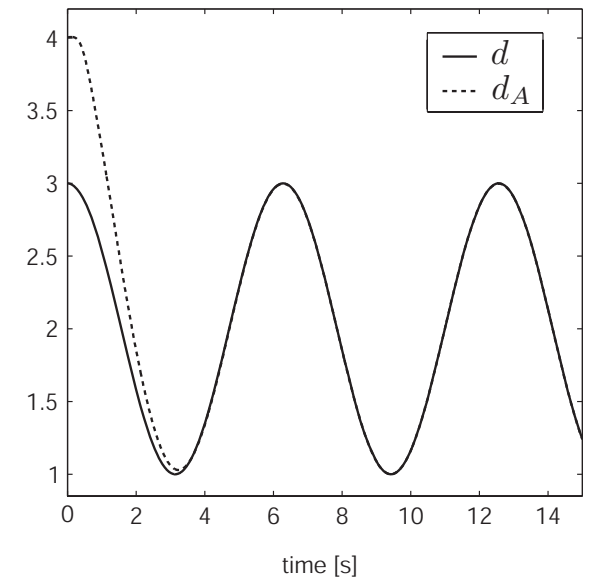

(c)

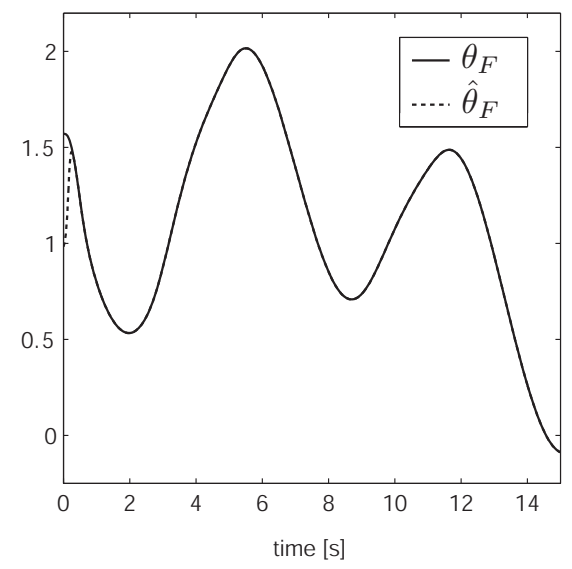

(e)

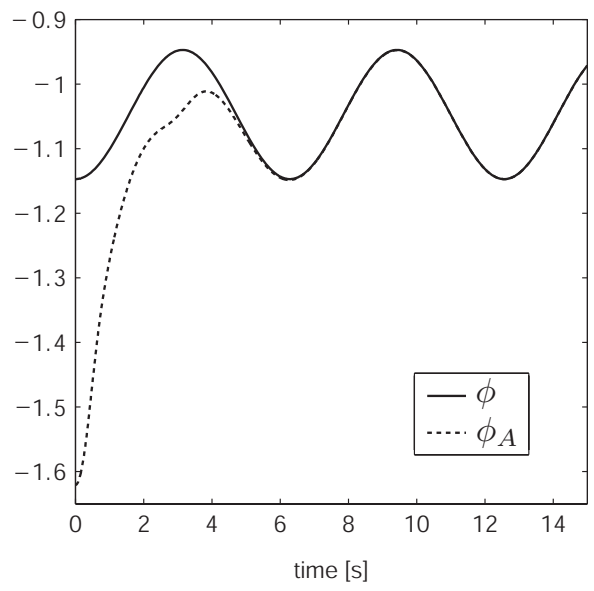

(d)

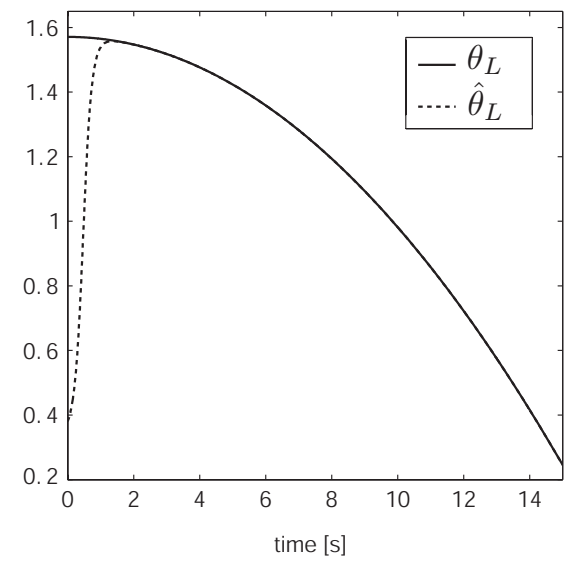

(f)

Fig. 3. Simulation I (ideal measurements): (a) Control inputs $v_{F}$ and $\omega_{F}$; (b) Sliding surfaces $s_{1}$ and $s_{2}$; (c) Desired distance $d$ and actual distance $d_{A}$; (d) Desired orientation $\phi$ and actual orientation $\phi_{A}$; (e) $\theta_{F}$ and $\hat{\theta}_{F}$; (f) $\theta_{L}$ and $\hat{\theta}_{L}$.

Fig. 2(a) shows the trajectory of the leader and the follower. In order to have a temporal reference in the figure the robots are drawn each second: the rectangles represent the tractor and the follower, while the small squares denote the trailer.
Fig. 2(b) shows the trajectory of the vehicles in the first 8 seconds of the simulation. In Fig. 3(a) the control inputs $v_{F}$ and $\omega_{F}$ are reported. In Fig. 3(b) the sliding surfaces $s_{1}$ and $s_{2}$ asymptotically converge to zero. Fig. 3(c) shows the 

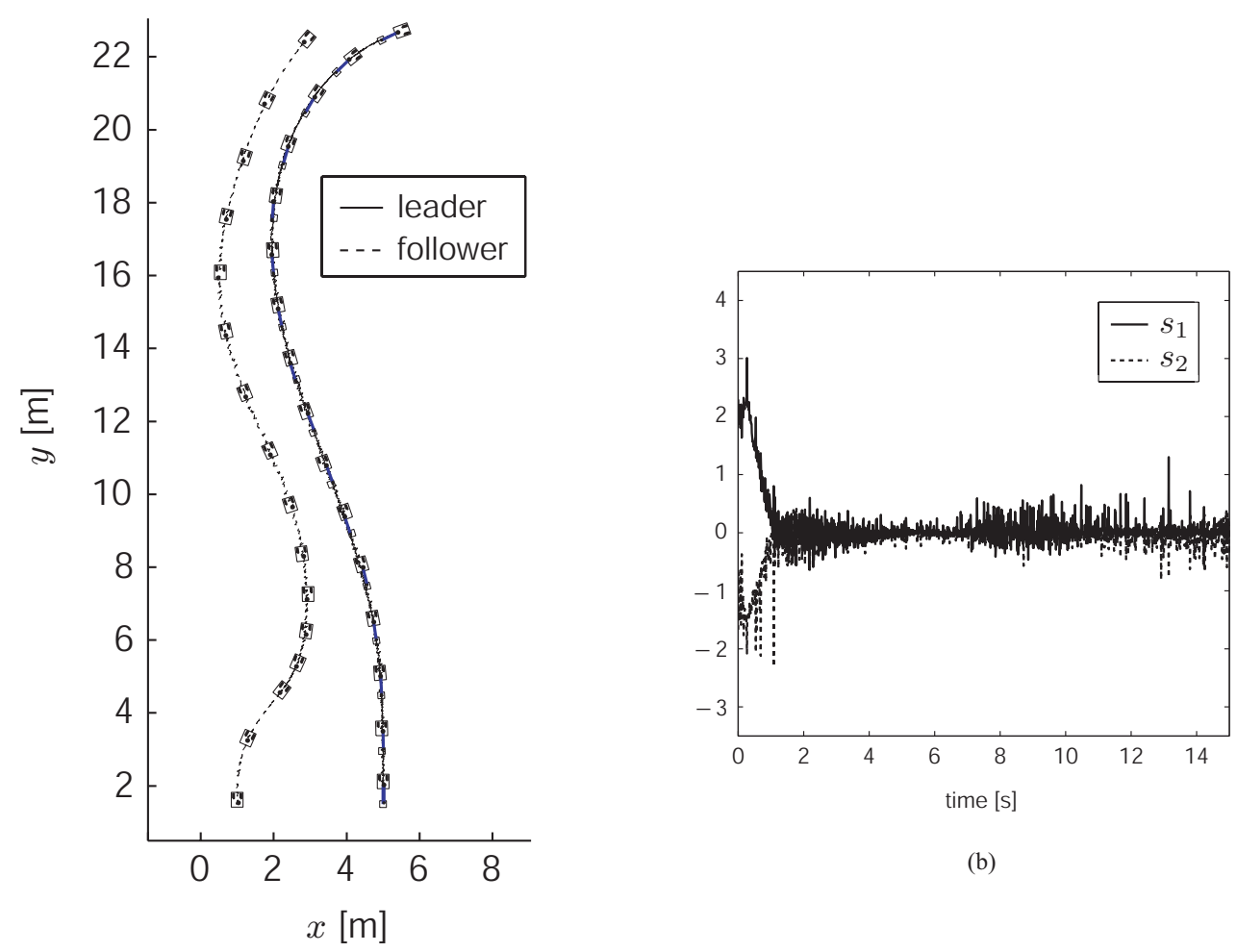

(a)
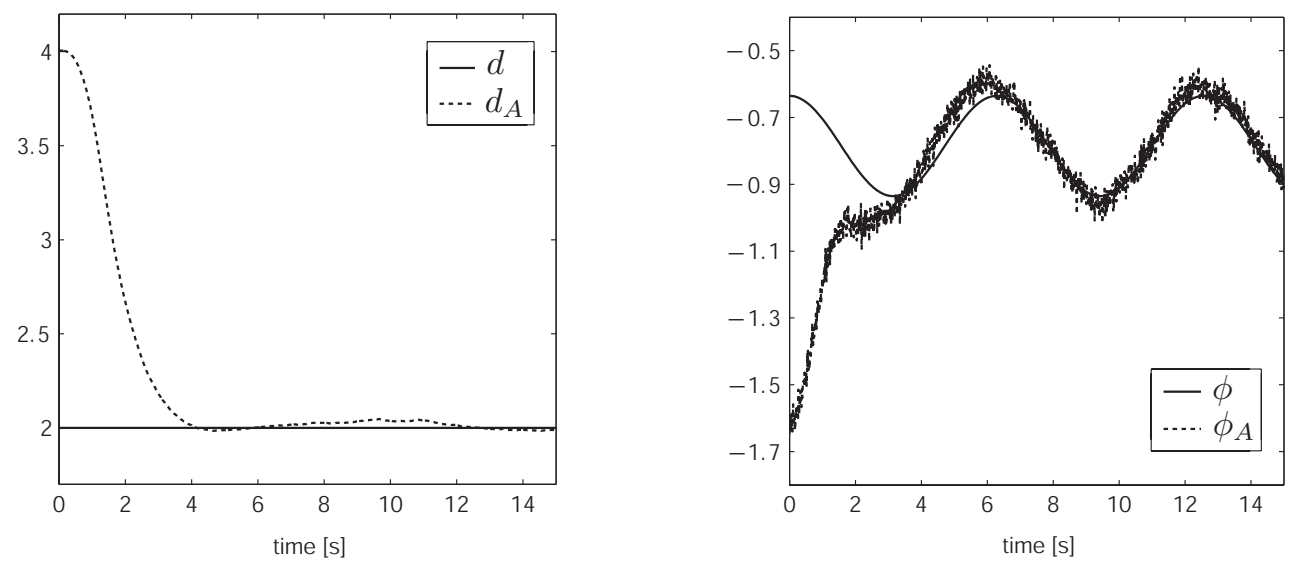

(c)

(d)

Fig. 4. Simulation II (noisy measurements): (a) Trajectory of the leader and the follower; (b) Sliding surfaces $s_{1}$ and $s_{2}$; (c) Desired distance $d$ and actual distance $d_{A}$; (d) Desired orientation $\phi$ and actual orientation $\phi_{A}$.

desired distance $d$ and the actual distance

\begin{tabular}{|c||c|c|}
\hline & Simulation I & Simulation II \\
\hline$v_{L}(t)[\mathrm{m} / \mathrm{s}]$ & 1.5 & 1.5 \\
\hline$\omega_{L}(t)[\mathrm{rad} / \mathrm{s}]$ & $-\pi / 2000$ & $\Omega(t)$ \\
\hline$d(t)[\mathrm{m}]$ & $\cos (t)+2$ & 2 \\
\hline$\phi(t)[\mathrm{rad}]$ & $-0.1 \cos (t)-\pi / 3$ & $0.15 \cos (t)-\pi / 4$ \\
\hline
\end{tabular}

$$
d_{A}=\left\|\begin{array}{c}
x_{L}-\ell_{2} \cos \eta_{L}-x_{F}-\ell_{1} \cos \theta_{F} \\
y_{L}-\ell_{2} \sin \eta_{L}-y_{F}-\ell_{1} \sin \theta_{F}
\end{array}\right\|
$$

Analogously, Fig. 3(d) shows the desired orientation $\phi$ and the actual orientation

TABLE I

LEADER'S VELOCITY AND DESIRED FUNCTIONS IN THE SIMULATIONS.

$$
\phi_{A}=\arctan \left(\frac{y_{L}-\ell_{2} \sin \eta_{L}-y_{F}-\ell_{1} \sin \theta_{F}}{x_{L}-\ell_{2} \cos \eta_{L}-x_{F}-\ell_{1} \cos \theta_{F}}\right)-\theta_{F}-\alpha_{F}
$$


$d_{A}, \phi_{A}$ and $d, \phi$ coincide after about 5 seconds. Finally, Figs. 3(e)-(f) show the time histories of $\theta_{F}, \hat{\theta}_{F}$ and $\theta_{L}, \hat{\theta}_{L}$.

The results of Simulation II are given in Fig. 4. Fig. 4(a) shows the trajectory of the leader and the follower, Fig. 4(b) the sliding surfaces and Figs. 4(c)-(d) the desired and actual distance and orientation. In spite of the noisy measurements, as in Simulation I, $d_{A}, \phi_{A}$ converge to $d, \phi$ and the sliding surfaces converge to zero. The control inputs $v_{F}, \omega_{F}$ and the time histories of $\theta_{F}, \hat{\theta}_{F}$ and $\theta_{L}, \hat{\theta}_{L}$ are not shown for lack of space. From the simulation experiments we observed that the estimate $\hat{\theta}_{F}$ (and analogously $\hat{\theta}_{L}$ ) is much more sensitive to position than angular disturbances.

\section{CONCLUSION}

In this paper we propose a new leader-follower formation of nonholonomic mobile robots. The follower is a car and the leader is an articulated vehicle, a tractor pulling a trailer. The desired formation, defined by two parameters (a distance and an orientation function) is allowed to vary in time. A sliding mode formation tracking control scheme and nonlinear observers for the estimation of the attitude angles of the follower and the tractor, are designed. These observers are based on the invariant manifold technique recently proposed in [15]. The effectiveness of the proposed designs has been validated via simulation experiments.

Future research lines include the experimental validation of our control scheme and the extension of our results to vehicles with more involved kinematics (e.g. the fire truck model [3] could be considered for the leader). For the sake of simplicity in the present paper a single-leader, single-follower formation has been considered. Future investigations will cover the more general case of multi-leader/multi-follower formations (see, e.g. [6]).

\section{ACKNOWLEDGMENTS}

The authors would like to thank the anonymous reviewers and conference attendees for their useful suggestions and constructive comments to improve the quality of paper.

\section{REFERENCES}

[1] J.G. Bender. An overview of systems studies of automated highway systems. IEEE Transactions on Vehicular Technology, 40(1):82-99, 1991.

[2] R. Burns, C.A. McLaughlin, J. Leitner, and M. Martin. TechSat21: Formation Design, Control and Simulation. In Proc. IEEE Aerospace Conference, volume 7, pages 19-25, 2000.

[3] L.G. Bushnell, D.M. Tilbury, and S.S. Sastry. Steering Three-Input Nonholonomic Systems: The Fire Truck Example. International Journal of Robotics Research, 14(4):366-381, 1995.

[4] L.E. Buzogany, M. Pachter, and J.J. D'Azzo. Automated Control of Aircraft in Formation Flight. In Proc. AIAA Guidance, Navigation, and Control Conference, pages 1349-1370, 1993.

[5] D. Chwa. Sliding-Mode Tracking Control of Nonholonomic Wheeled Mobile Robots in Polar Coordinates. IEEE Transactions on Control Systems Technology, 12(4):637-644, 2004.

[6] L. Consolini, F. Morbidi, D. Prattichizzo, and M. Tosques. A Geometric Characterization of Leader-Follower Formation Control. In Proc. IEEE International Conference on Robotics and Automation, pages 23972402, 2007.
[7] A.K. Das, R. Fierro, V. Kumar, J.P. Ostrowsky, J. Spletzer, and C. Taylor. A Vision-Based Formation Control Framework. IEEE Transaction on Robotics and Automation, 18(5):813-825, 2002.

[8] R.A. DeCarlo, S.H. Zak, and G.P. Matthews. Variable Structure Control of Nonlinear Multivariable Systems: A Tutorial. Proc. of the IEEE, 76(3):212-232, 1988

[9] K.D. Do and J. Pan. Nonlinear formation control of unicycle-type mobile robots. Robotics and Autonomous Systems, 55:191-204, 2007.

[10] D.B. Edwards, T.A. Bean, D.L. Odell, and M.J. Anderson. A leaderfollower algorithm for multiple AUV formations. In Proc. IEEE/OES Autonomous Underwater Vehicles, pages 40-46, 2004.

[11] J.A. Fax and R.M. Murray. Information Flow and Cooperative Control of Vehicle Formations. IEEE Transactions on Automatic Control, 49(9):1465-1476, 2004.

[12] T.I. Fossen. Guidance and Control of Ocean Vehicles. John Wiley and Sons, New York, 1994.

[13] V. Gazi. Swarm Aggregations Using Artificial Potentials and SlidingMode Control. IEEE Transactions on Robotics, 21(6):1208-1214, 2005.

[14] J.Y. Hung, W. Gao, and J.C. Hung. Variable Structure Control: A Survey. IEEE Transactions on Industrial Electronics, 40(1):2-22, 1993.

[15] D. Karagiannis and A. Astolfi. Nonlinear observer design using invariant manifolds and applications. In Proc. 44th IEEE Conference on Decision and Control, pages 7775-7780, 2005.

[16] T.J. Koo and S.M. Shahruz. Formation of a group of unmanned aerial vehicles (UAVs). In Proc. American Control Conference, volume 1, pages $69-74,2001$.

[17] X. Li, J. Xiao, and Z. Cai. Backstepping based multiple mobile robots formation control. In Proc. IEEE/RSJ International Conference on Intelligent Robots and Systems, pages 887-892, 2005.

[18] Z. Lin, B.A. Francis, and M. Maggiore. Necessary and Sufficient Graphical Conditions for Formation Control of Unicycles. IEEE Transactions on Automatic Control, 50(1):121-127, 2005.

[19] G.L. Mariottini, F. Morbidi, D. Prattichizzo, G.J. Pappas, and K. Daniilidis. Leader-Follower Formations: Uncalibrated Vision-Based Localization and Control. In Proc. IEEE International Conference on Robotics and Automation, pages 2403-2408, 2007.

[20] G.L. Mariottini, G.J. Pappas, D. Prattichizzo, and K. Daniilidis. Visionbased Localization of Leader-Follower Formations. In Proc. 44th IEEE Conference on Decision and Control, pages 635-640, 2005.

[21] W. Perruquetti and J.P. Barbot. Sliding mode control in engineering. Control Engineering Series. Marcel Dekker Inc., 2002.

[22] M. Pham and D. Wang. A Unified Nonlinear Controller for a Platoon of Car-Like Vehicles. In Proc. American Control Conference, volume 3, pages 2350-2355, 2004.

[23] J. Sànchez and R. Fierro. Sliding Mode Control for Robot Formations. In Proc. IEEE International Symposium on Intelligent Control, pages 438-443, 2003.

[24] H. Schaub, S.R. Vadali, J.L. Junkins, and K.T. Alfriend. Spacecraft Formation Flying Control Using Mean Orbit Elements. Journal of the Astronautical Sciences, 48(1):69-87, 2000.

[25] J. Shao, G. Xie, J. Yu, and L. Wang. Leader-Following Formation Control of Multiple Mobile Robots. In Proc. IEEE/RSJ International Symposium on Intelligent Control, pages 808-813, 2005.

[26] D. Swaroop and J.K. Hedrick. Constant spacing strategies for platooning in Automated Highway systems. Journal of dynamic systems, measurement, and control, 121(3):462-470, 1999.

[27] V.I. Utkin. Variable structure systems with sliding modes. IEEE Transactions on Automatic Control, 22(2):212-222, 1977.

[28] V.I. Utkin. Sliding Mode Control Design Principles and Applications to Electric Drives. IEEE Transactions on Industrial Electronics, 40(1):23-36, 1993.

[29] R. Vidal, O. Shakernia, and S. Sastry. Following the Flock: Distributed Formation Control with Omnidirectional Vision-Based Motion Segmentation and Visual Servoing. IEEE Robotics and Automation Magazine, 11(4):14-20, 2004.

[30] E. Yang, D. Gu, and H. Hu. Nonsingular formation control of cooperative mobile robots via feedback linearization. In Proc. IEEE/RSJ International Conference on Intelligent Robots and Systems, pages 826831,2005

[31] J.-M. Yang and J.-H. Kim. Sliding Mode Control for Trajectory Tracking of Nonholonomic Wheeled Mobile Robots. IEEE Transactions on Robotics and Automation, 15(3):578-587, 1999. 